\title{
Regression of flow-induced pulmonary arterial vasculopathy after flow correction in piglets
}

Olaf Mercier, MD, Edouard Sage, MD, Marc de Perrot, MD, Ly Tu, BSc, Elisabeth Marcos, BSc, Benoît Decante, MSc, Bruno Baudet, BSc, Philippe Hervé, MD, Philippe Dartevelle, MD, Saadia Eddahibi, PhD, and Elie Fadel, MD

\begin{abstract}
Objectives: Chronic thromboembolic pulmonary hypertension is due to partial obstruction of the pulmonary arterial bed and may resolve after pulmonary thromboendarterectomy. Persistent pulmonary hypertension, the main complication after pulmonary thromboendarterectomy, may reflect vessel alterations induced by high flow in unobstructed lung territories. The aim of this study was to determine whether correcting high flow led to reversal of the vasculopathy in piglets.
\end{abstract}

\begin{abstract}
Methods: The effects of high pulmonary blood flow were investigated 5 weeks after creation of an aortopulmonary shunt $(\mathrm{n}=10)$, and reversibility of vessel disease was evaluated at 1 week $(\mathrm{n}=10)$ and 5 weeks after shunt closure $(n=10)$, compared to sham-operated animals $(n=10)$. Hemodynamic variables, pulmonary artery reactivity, and morphometry were recorded. We also investigated the endothelin, angiopoietin, and nitric oxide synthase pathways.

Results: High flow increased medial thickness in distal pulmonary arteries $(55.6 \% \pm 1.2 \%$ vs $35.9 \% \pm 0.8 \%$; $P<.0001$ ) owing to an increase of smooth muscle cell proliferation (proliferating cell nuclear antigen labeling). The endothelium-dependent relaxation was altered $(P<.05)$. This phenomenon was associated to an overexpression of endothelin-1, endothelin-A, angiopoietin 1, angiopoietin 2, and Tie-2 $(P<.05)$. After 1 week of shunt closure, all overexpressed genes returned to control values, the proliferation of smooth muscle cells stopped ,and smooth muscle cell apoptosis increased (terminal deoxynucleotidyl transferase-mediated dUTP nick end labeling), preceding the normalization of the wall thickness hypertrophy and the pulmonary artery vasoreactivity observed at 5 weeks after shunt closure.
\end{abstract}

Conclusion: These results demonstrate that endothelin- 1 and angiopoietin pathways are involved in vasculopathy development and may be important therapeutic targets for preventing persistent pulmonary hypertension after pulmonary thromboendarterectomy.

Chronic thromboembolic pulmonary hypertension (CTEPH) is a life-threatening disease with an overall 5-year survival of $10 \%$ in patients whose mean pulmonary artery (PA) pressure exceeds $50 \mathrm{~mm} \mathrm{Hg.}{ }^{1} \mathrm{CTEPH}$ is more common than previously thought and may occur in up to $3.8 \%$ of patients with acute pulmonary embolism. ${ }^{2}$ Organized thrombotic material causing chronic vascular obstruction is the pathogenic mechanism, and pulmonary thromboendarterectomy (PTE) provides long-term hemodynamic and functional benefits and increases life expectancy. ${ }^{3-5}$ However, PTE is associated with $5 \%$ to $23 \%$ mortality, related chiefly to reperfusion pulmonary edema and persistent pulmonary hypertension $(\mathrm{PH})$ despite removal of the thrombotic material. ${ }^{3-5}$

From the Laboratoire de Chirurgie Expérimentale UPRES-EA 2705, Uninersité Paris XI Hôpital Marie Lannelongue, Le Plessis-Robinson, France.

This work was supported by a grant from ADETEC, France.

Received for publication Feb 10, 2008; revisions received June 23, 2008; accepted for publication July 26, 2008.

Address for reprints: Olaf Mercier, MD, Laboratoire de Chirurgie Expérimentale UPRES-EA 2705, Hôpital Marie Lannelongue, 133, Avenue de la Résistance, 92350 Le Plessis-Robinson, France (E-mail: o.mercier@ccml.fr).

J Thorac Cardiovasc Surg 2009;137:1538-46

0022-5223/\$36.00

Copyright (c) 2009 by The American Association for Thoracic Surgery

doi:10.1016/j.jtcvs.2008.07.069
Two vascular territories can be distinguished in the lung after pulmonary thromboembolism: the obstructed territory characterized by chronic ischemia and the unobstructed territory exposed to chronic high blood flow resulting from redistribution of the cardiac output. ${ }^{6,7}$ This unobstructed territory contributes to the elevated pulmonary vascular resistance, ${ }^{8}$ inasmuch as chronic high flow causes distal arteriopathy with vascular remodeling ${ }^{9,10}$ similar to that found in primary $\mathrm{PH} .{ }^{11-15}$

Pulmonary thromboendarterectomy in patients with high pulmonary vascular resistance is associated with high rates of mortality and postoperative persistent PH. Preoperative prostacyclin infusion, which induces PA vasodilatation and inhibits vascular remodeling, has been found to decrease post-PTE mortality in patients with preoperative pulmonary resistance values exceeding 1200 dyne $\cdot \mathrm{s} \cdot \mathrm{cm}^{5} \cdot{ }^{16}$ These findings support the hypothesis that the distal arteriopathy induced by chronic high blood flow in unobstructed territories may be the cause of persistent PH after PTE.

Knowledge of the pathobiological mechanisms underlying $\mathrm{PH}$ has significantly improved over the past decade. Three pathways seem to play a key role in the development of PH: endothelin, angiopoietin, and nitric oxide synthase (NOS). ${ }^{17-19}$ The endothelial dysfunction seen in PH is associated with an imbalance between endothelium-derived 


\section{Abbreviations and Acronyms \\ $\mathrm{CTEPH}=$ chronic thromboembolic pulmonary hypertension \\ eNOS = endothelial nitric oxide synthase \\ ET $=$ endothelin \\ iNOS = inducible nitric oxide synthase \\ NOS = nitric oxide synthase \\ PA = pulmonary artery \\ PBS = phosphate-buffered saline \\ PCNA $=$ proliferating cell nuclear antigen \\ $\mathrm{PH} \quad=$ pulmonary hypertension \\ PTE = pulmonary thromboendarterectomy \\ SMC $=$ smooth muscle cell \\ TUNEL $=$ terminal deoxynucleotidyl transferase- mediated dUTP nick end labeling}

vasoconstrictor/mitogenic factors and vasodilatator/nonmitogenic factors. However, few studies to understand mechanisms underlying CTEPH had been published. Angiopoietin-1 expression was found to be increased in patients with CTEPH, as in various other forms of $\mathrm{PH} .{ }^{19}$

We studied the effects of chronic high blood flow to the lungs in an experimental animal model of aortopulmonary shunting. We determined whether the effects of high pulmonary blood flow resolved after restoring normal blood flow to simulate the effects of PTE. We analyzed nitric oxide, endothelin, and angiopoietin pathways to understand pathophysiologic mechanisms.

\section{MATERIALS AND METHODS}

Forty Large White piglets weighing $21 \pm 3 \mathrm{~kg}$ were used. The study complied with the "Principles of Laboratory Animal Care," developed by the National Society for Medical Research, and the "Guide for the Care and Use of Laboratory Animals," written by the Institute of Laboratory Animal Resources and published by the National Institutes of Health (publication No.86-23, revised in 1985).

\section{Experimental Design}

The piglets were randomly allocated to four groups $(\mathrm{n}=10$ in each group). The studies were done 5 weeks after performing an aortopulmonary shunt to increase blood flow into PAs (shunt-open group), 1 week (1-week shunt-closed group) and 5 weeks (5-week shunt-closed group) after closure of this shunt, and 5 weeks after PA dissection without shunt in the sham group.

\section{Surgical Procedures}

Aortopulmonary shunt. An aortopulmonary shunt was performed as previously described by Rendas, Lennox, and Reid. ${ }^{20}$ An 8-mm diameter polytetrafluoroethylene bypass was implanted between the ascending aorta and the main PA. Fractionated heparin was given until harvesting of the lungs or closure of the shunt to avoid postoperative occlusion of the shunt. Aortopulmonary shunt closure. The midline sternotomy was reopened. After systemic heparinization, the aorta and main PA were partially clamped on each side of the bypass, which was divided and sutured.

\section{Hemodynamic Measurements and Lung Harvesting}

Pulmonary hemodynamics were measured before the animals were killed. Aortic blood flow was measured with a flow probe (Transonic Systems, Inc, Ithaca, NY) placed on the origin of the aorta upstream from the aortopulmonary shunt. The pulmonary and systemic flow values were measured downstream from the shunt.

Pressures and blood gases were measured by direct puncture downstream from the aortopulmonary shunt. Then, the animal was exsanguinated and the lungs rapidly removed from the chest. Patency of the shunt was assessed by direct visual inspection. Biopsy specimens weighing 300 to 500 $\mathrm{mg}$ from the left upper lobe were snap-frozen in liquid nitrogen and stored at $-70^{\circ} \mathrm{C}$ or left to fix in $4 \%$ paraformaldehyde solution.

\section{Light Microscopy and Morphometry}

Fixed lung sections were processed by standard histologic techniques and embedded in paraffin. We sought to identify 30 to 40 arteries of less than $200 \mu \mathrm{m}$ in diameter in each piglet. Medial thickness (MT) was calculated as followes:

$$
\% \mathrm{MT}=(\mathrm{ED}-\mathrm{ID}) \times 100 / \mathrm{ED}
$$

Where $\mathrm{ED}=$ external diameter and $\mathrm{ID}=$ internal diameter.

\section{Evaluation of In Situ PA-Smooth Muscle Cell (SMC) Death and Proliferation and Collagen Accumulation}

To assess the PA-SMC proliferation, we evaluated the proliferating cell nuclear antigen (PCNA). Tissue sections were deparaffinized in xylene, followed by treatment with a graded series of alcohol washes, rehydratation in phosphate-buffered saline (PBS) (pH 7.5), and then incubated with target retrieval solution (Dako, Trappes, France) into a water bath at $90^{\circ} \mathrm{C}$ for 20 minutes. Endogenous peroxidase activity was blocked with $\mathrm{H}_{2} \mathrm{O}_{2}$ in PBS ( $3 \% \mathrm{vol} / \mathrm{vol})$ for 5 minutes. Slides were then washed in PBS and incubated for 30 minutes in a protein-blocking solution consisting of PBS supplemented with $3 \%$ bovine serum albumin. The slides were subsequently incubated for 30 minutes with anti-PCNA mouse monoclonal antibody (PC-10, 1:200, Dako, Trappes, France). Antibodies were washed off and the slides were processed with the alkaline phosphatase LSAB+ systemHRP detection kit (DAKO, Carpinteria, Calif). Brown color was generated by using a diaminobenzidine substrate and nuclei were counterstained with hematoxylin.

Detection of cells undergoing apoptosis was evaluated by the ApopTag Red In Situ Apoptosis Detection Kit (Qbiogene, Illkirch, France), as specified by the manufacturer.

So that collagen accumulation could be assessed, the paraffin-embedded sections were deparaffinized and stained with Masson trichrome stain.

\section{Assessment of PA reactivity}

Pulmonary artery rings were studied as previously described. ${ }^{21}$ Endothelin-1 (ET-1), sodium nitroprusside, acetylcholine hydrochloride, and indomethacin were purchased from Sigma Chemical Company (St Louis, Mo)

\section{Real-Time Quantification by Polymerase Chain Reaction Assay}

Real-time polymerase chain reaction assay was conducted as previously described. ${ }^{12}$ Except for the report gene 18S, angiopoietins, and Tie-2, all the primers (ET-1, ETA, ETB, endothelial NOS [eNOS], inducible NOS [iNOS]) were specific porcine primers previously described by Rondelet and associates. ${ }^{12}$ Primers for $18 \mathrm{~S}$ ribonucleic acid, angiopoietins and Tie-2 had been used previously in our laboratory for human experimentation and were designed on Primer Express software (Applied Biosystems, Foster City, Calif).

\section{Assay of Lung Phosphodiesterase 5 Activity}

Phosphodiesterase 5 assays were carried out from frozen lung tissue according to established procedures. ${ }^{22}$ 
TABLE 1. Changes in hemodynamic variables and blood gas levels induced by opening and by closing an aortopulmonary shunt

\begin{tabular}{lcccc}
\hline & & & \multicolumn{2}{c}{ Shunt closed } \\
\cline { 3 - 5 } & Sham & Shunt open & One week & Five weeks \\
\hline Weight $(\mathrm{kg})$ & $24 \pm 4$ & $28 \pm 3$ & $46 \pm 8$ & $40 \pm 6$ \\
$\mathrm{HR}(\mathrm{beats} / \mathrm{min})$ & $98 \pm 4$ & $89 \pm 3$ & $89 \pm 9$ & $89 \pm 9$ \\
$\mathrm{Q}\left(\mathrm{L} \cdot \mathrm{min}^{-1}\right)$ & $2 \pm 0.14$ & $3.3 \pm 0.3^{*}$ & $2.14 \pm 0.6 \ddagger$ & $51 \pm 3$ \\
$\mathrm{Mean} \mathrm{SAP}(\mathrm{mm} \mathrm{Hg})$ & $55 \pm 3$ & $51 \pm 4$ & $10.2 \pm 2 \ddagger$ & $57 \pm 4$ \\
$\mathrm{Mean} \mathrm{PAP}(\mathrm{mm} \mathrm{Hg})$ & $10.1 \pm 0.5$ & $15.7 \pm 1.2^{*}$ & $282 \pm 36$ & $10.2 \pm 1.2 \dagger$ \\
$\mathrm{PaO}_{2}(\mathrm{~mm} \mathrm{Hg})$ & $336.65 \pm 18$ & $262 \pm 19^{*}$ & $38.5 \pm 3 \ddagger$ & $367 \pm 20 \dagger$ \\
$\mathrm{PA} \mathrm{Po}_{2}(\mathrm{~mm} \mathrm{Hg})$ & $41.7 \pm 2.6$ & $47.2 \pm 2.7$ & $36.5 \pm 1.6 \dagger$ \\
\hline
\end{tabular}

$\mathrm{HR}$, Heart rate; $Q$, cardiac outflow; $\mathrm{SAP}$, systemic arterial pressure; $\mathrm{PAP}$, pulmonary arterial pressure; $\mathrm{PaO}_{2}$, systemic $\mathrm{Po}_{2} ; \mathrm{PAPO}_{2}$, pulmonary artery $\mathrm{Po}_{2}$. ${ }^{*} \mathrm{P}<.05$ sham group vs shunt-open group. $\dagger P<.05$ shunt-open group vs 5-week shunt-closed group. $\ddagger P<.05$ shunt-open group vs 1 -week shunt-closed group.

\section{Statistical Analysis}

All results are reported as means \pm SEM. The Kolmogorov-Smirnov test was applied to test for normality of the distribution of each variable. Oneway analysis of covariance was performed using the weight of the piglets as the covariate, followed by Fisher's test for between-group comparisons. All statistical analyses were performed using Statview IV software (Abacus Concepts, Berkeley, Calif).

\section{RESULTS}

\section{Effect of the Aortopulmonary Shunt on} Hemodynamic Variables

None of the piglets died during or after the surgical procedures. The animals in the shunt-closed groups were kept longer than animals in the other two groups and therefore had a greater mean weight gain. Aortography performed before the animals were killed showed that the shunt was patent in piglets from the shunt-open group and occluded in piglets from the shunt-closed groups. As summarized in Table 1, cardiac output and mean PA pressure were elevated in the shunt-open group. Heart rate and mean arterial pressure were not significantly changed after 5 weeks of shunting. Cardiac output and mean PA pressure returned to sham values 1 and 5 weeks after shunt closure, and no changes were found in heart rate and mean arterial pressure. As expected $\mathrm{PA} \mathrm{PO}_{2}$ was increased in the shunt-open group compared with the sham group; $\mathrm{PA} \mathrm{PO}_{2}$ in the shunt-closed groups was not significantly different from that in the sham group. The pulmonary/systemic flow values were similar in the shunt-open groups.

\section{Pulmonary Vascular Remodeling}

In the sham group, medial wall thickness of PAs less than $200 \mu \mathrm{m}$ in diameter was $35.9 \% \pm 0.8 \%$. Five weeks after shunt creation, distal vascular remodeling was visible as a significant increase in medial wall thickness when compared with sham values $(55.6 \% \pm 1.2 \%$ vs $35.9 \% \pm$ $0.8 \% ; P<.001)$. Decrease in distal medial thickness followed closure of the shunt at 1 week $(50 \% \pm 1.4 \%$ vs $55.6 \% \pm 1.2 \% ; P=.062)$ and 5 weeks $(40.9 \% \pm 1 \%$ vs $55.6 \% \pm 1.2 \% ; P<.001)$, respectively. Medial thickness values returned to near sham values after 5 weeks of closure of the shunt (Figures 1A and 1B).
Proliferating cell nuclear antigen labeling demonstrated a proliferation of SMCs in the distal PA wall in shunted animals. As early as 1 week after shunt closure, the number of PCNA-positive cells decreased markedly and returned to sham values $(22.4 \% \pm 15 \%$ vs $6 \% \pm 9 \% ; P<.05)$. Conversely, the number of apoptotic cells as detected by terminal deoxynucleotidyl transferase-mediated dUTP nick end labeling (TUNEL) in PA walls in the shunted group was similar to that in the sham group $(6.6 \% \pm 6 \%$ vs $9.2 \%$ $\pm 10 \% ; P=$ not significant), peaked at 1 week after the shunt closure, and then returned toward sham values at 5 weeks after shunt removal $(23.1 \% \pm 18 \%$ vs $9.5 \% \pm$ $9 \% ; P<.05)$. Collagen accumulation around the PAs was not different between groups (Figures 2A and 2B).

\section{Pulmonary Vascular Reactivity and Endothelial Function (Figure 3)}

Contraction of PA rings in response to phenylephrine was not significantly different in the four groups. Incubation of arteries with increasing doses of ET-1 induced dose-dependent contraction that was similar in the shunt-open, 1-week shunt-closed, and sham groups. In the 5-week shunt-closed group, however, maximal contraction induced by ET-1 was significantly lower than in the sham group (1722 mg vs 2380 mg; $P=.019$ ), although median effective concentration values were not significantly different.

The relaxation response to sodium nitroprusside was not affected by aortopulmonary shunt creation or occlusion. Aortopulmonary shunting was followed by a decrease in maximal relaxation to acetylcholine $(51.9 \% \pm 5.42 \%$ vs $66.8 \% \pm 6.6 \% ; P=.045)$. Maximal relaxation to acetylcholine was not restored 1 week after closure of the shunt $(52.1 \% \pm 4.62 \%$ vs $51.9 \% \pm 5.42 \% ; P=.97)$ but returned to normal values at 5 weeks $(67.6 \% \pm 5.4 \%$ vs $66.8 \% \pm$ $6.6 \% ; P=.93)$. Median effective concentration values were unchanged.

\section{Expression of Genes Involved in the ET-1, NOS, and Angiopoietin-1 Pathways}

Increased lung blood flow induced an increase in ET-1, ETA, angiopoietin-1, angiopoietin-2, and Tie-2 mRNA 

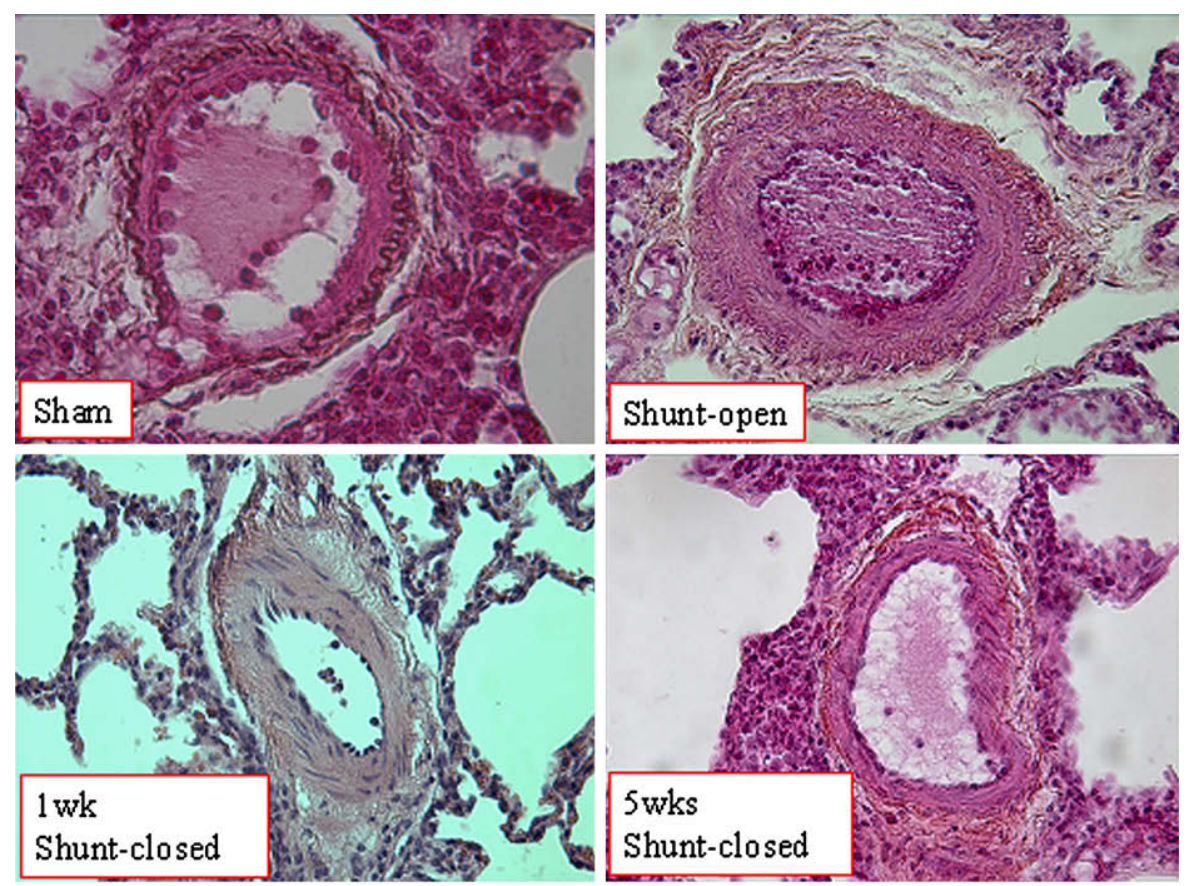

A

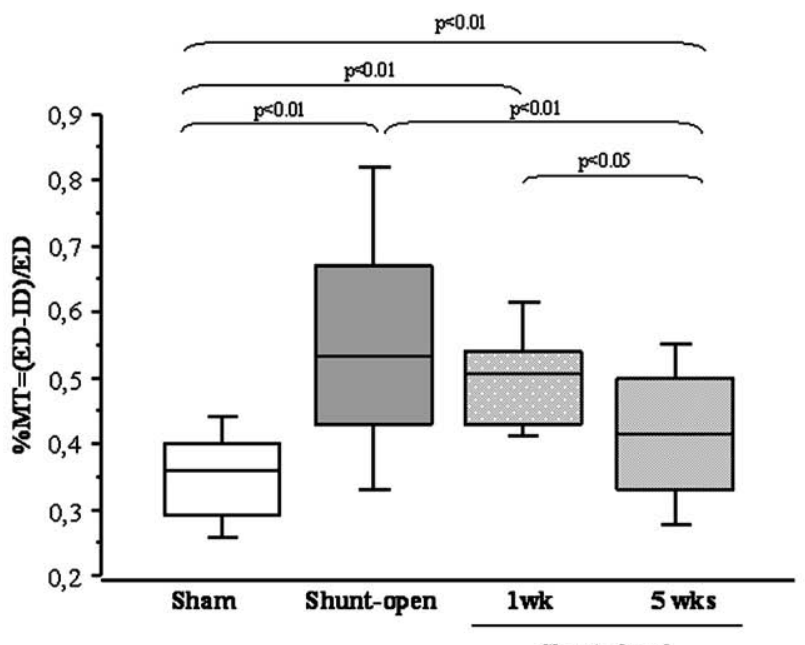

B

Shunt-closed

FIGURE 1. A, Morphometry $(\times 400)$ of pulmonary arteries of less than $150-\mu \mathrm{m}$ diameter in the sham group, the shunt-open group, and the shunt-closed groups. The percentage of medial thickness was the highest in the shunt-open group $(P<.05)$. Medial thickness started to decrease after 1 week of shunt closure and raised a decrease of $75 \%$ after 5 weeks of shunt closure $(P<.001)$. B, Box plot of the percentage of medial thickness of small pulmonary arteries $(<150 \mu \mathrm{m})$ in the sham $(35.9 \% \pm 0.8 \%)$, shunt-open $(55.6 \% \pm 1.2 \%), 1$-week shunt-closed $(48.7 \% \pm 1 \%)$, and 5 -week shunt-closed $(40.9 \% \pm 1 \%)$ groups. $M T$, Medial thickness; $E D$, external diameter; $I D$, internal diameter.

levels, without changes in steady-state levels of ETB or iNOS (Figures 4 and 5). For eNOS, mRNA levels were higher in the shunt-open group than in the sham group, whereas protein contents in lung tissue were not different (data not shown).

One week after shunt closure, ET-1, ETA, angiopoietin-1, angiopoietin-2, and Tie-2 mRNA levels returned to normal values. The normalization of morphometric parameters as seen 5 weeks after the shunt closure was preceded by a decrease of all overexpressed gene mRNA.
Five weeks after closure of the aortopulmonary shunt, we observed an increase of ETB mRNA levels and a paradoxical increase of normalized ET-1 mRNA levels (Figure 4).

\section{Phosphodiesterase 5 Activity}

To determine whether the decrease in endothelium-dependent relaxation was related to an increase in phosphodiesterase 5 activity induced by high blood flow, we measured phosphodiesterase 5 activity in lung tissue from piglets in all 


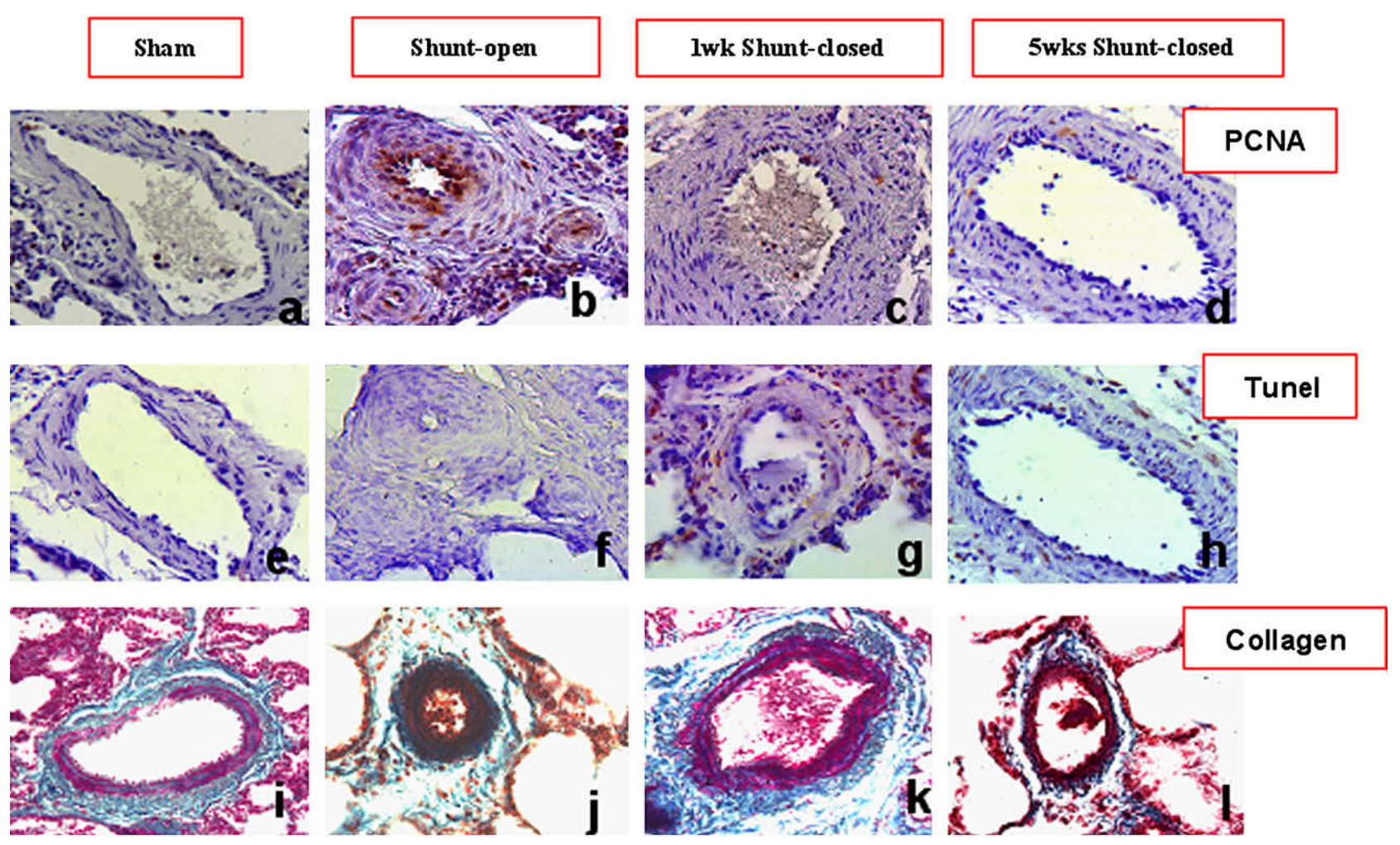

A
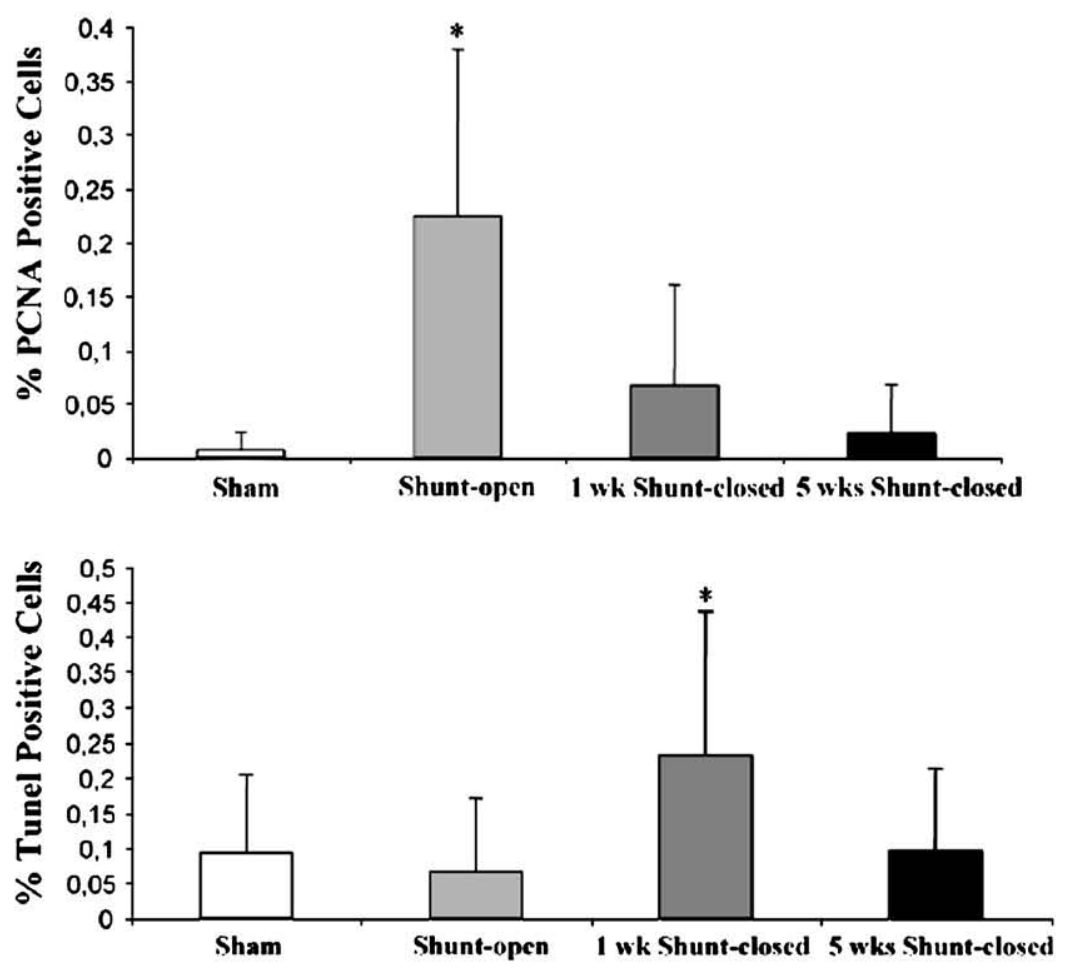

B $* \mathrm{p}<0,05$ 

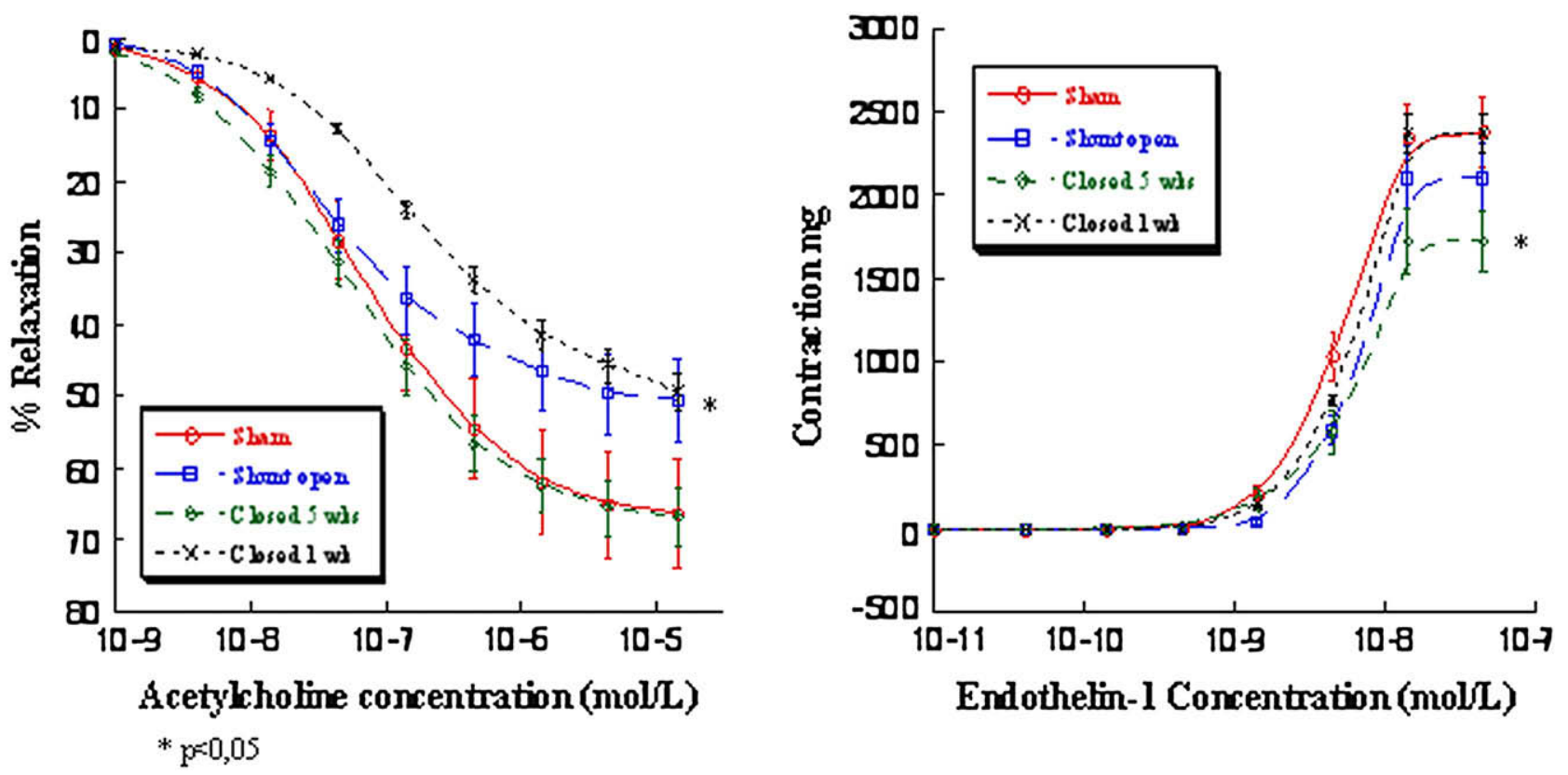

FIGURE 3. Vasoconstriction to endothelin-1 and relaxation to acetylcholine of PA rings from the sham group, the shunt-open group, and the shunt-closed groups. Aortopulmonary shunting induced a decrease in maximal relaxation to acetylcholine $(51.9 \% \pm 5.42 \%$ vs $66.8 \% \pm 6.6 \%$ : $P=.045)$. Five-week shunt closure was associated with recovery of the maximal relaxation to acetylcholine $(67.6 \% \pm 5.4 \%$ vs $66.8 \% \pm 6.6 \%$; $P=.93)$, whereas its remains unchanged 1 week after shunt closure. In the 5-weeks shunt-closed group, ET-1-induced maximal contraction was lower than in the sham group (1722 $\mathrm{mg}$ vs $2380 \mathrm{mg}, P=.019)$. $P A$, Pulmonary artery; ET-1, endothelin-1.

groups. Phosphodiesterase 5 activity was not affected by creation or closure of the shunt (data not shown).

\section{DISCUSSION}

This study showed that 5 weeks of aortopulmonary shunting induced pulmonary vasculopathy manifesting as medial thickness of the distal PAs owing to SMC proliferation, impaired endothelium-dependent vasorelaxation, and increase of ET-1, ETA, angiopoietin-1, angiopoietin-2, and Tie-2 expression in lung parenchyma. This study also demonstrates for the first time that after shunt closure, in the early phase (1 week), we observed a normalization of gene expression and later (5 weeks) a decrease of distal PA medial thickness, a normalization of endothelium-dependent vasorelaxation, and an increase of ETB expression.

After pulmonary embolism, the unobstructed lung territory is exposed to chronic high blood flow owing to redistribution of the cardiac output. ${ }^{6,7}$ This overflow induces a progressive increase of PA pressure and shear stress owing to a distal PA vasculopathy. We also speculate that the long-term improvement in pulmonary vascular resistance seen after PTE in humans may reflect resolution of flow-induced arterial disease. Similarly, in patients with severe CTEPH, extensive PA lesions induced by overflow may result in failure of PTE to lower pulmonary vascular resistance and, therefore, in increased postoperative morbidity and mortality. ${ }^{6-8}$ Our model of aortopulmonary shunting had been previously used as a model of lung disease associated with congenital heart disorder. ${ }^{12}$ Because it was not associated with chronic ischemic lung territories, this model neglected the potential effect of these territories on the nonischemic lung territories. However, this model adequately reproduces functional vascular changes seen in the unobstructed territories of patients with CTEPH. Indeed, as in humans, the aortopulmonary shunting induced a high pulmonary blood flow and increased PA pressure. To assess

FIGURE 2. A, Evaluation of in situ PA-SMCs death as assessed by TUNEL (e-h), PA-SMC proliferation by PCNA coloration (a-d), and collagen accumulation by Masson trichrome staining (i-1) in the shunt-open, 1-week shunt-closed, 5 -week shunt-closed, and sham groups $(\times 400)$. Positive cells in TUNEL and PCNA staining are colored in brown. Collagen is colored in blue by Masson trichrome staining. PA wall thickness in the shunt-open group is related to the proliferation of SMCs (b) without intravascular collagen accumulation (j). The decrease of PA medial thickness observed 5 weeks after the shunt closure started at 1 week with a decrease of PCNA-positive cells (c) and increased TUNEL-positive cells (g). PA, Pulmonary artery; SMC, smooth muscle cell; TUNEL, terminal deoxynucleotidyl transferase-mediated dUTP nick end labelling, PCNA, proliferating cell nuclear antigen. B, Percentage of PCNA- and TUNEL-positive cells in the wall of distal PAs for all groups of piglets. There is an SMC proliferation in the wall of distal PAs in the shunt-open group that stopped at the shunt closure. SMC apoptosis increased 1 week after the shunt closure and returned to normal value 5 weeks after the closure. $P A$, Pulmonary artery; SMC, smooth muscle cell; TUNEL, terminal deoxynucleotidyl transferase-mediated dUTP nick end labelling, $P C N A$, proliferating cell nuclear antigen. 


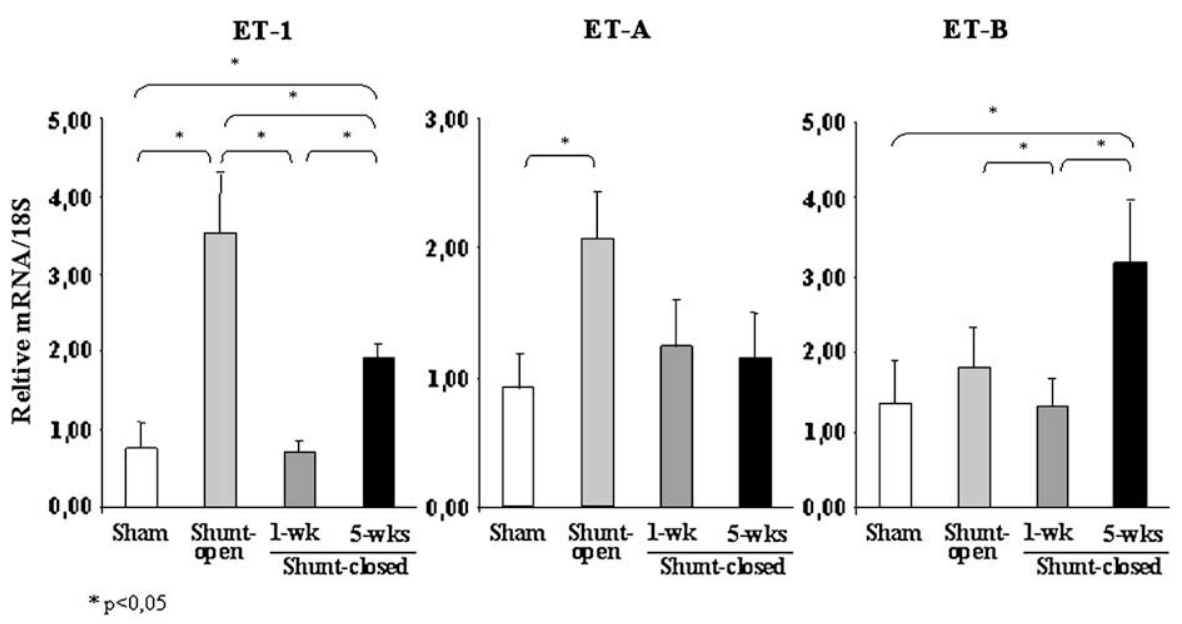

FIGURE 4. Quantification of endothelin-1 (ET-1) and its receptors (ETA and ETB) mRNAs in lung parenchyma by reverse-transcriptase polymerase chain reaction. High flow in the lungs induced increases in ET-1 and ETA mRNAs but did not change steady-state level of ETB. Shunt closure was followed by early (1 week) decrease in ET-1 and ETA mRNA levels. ETB mRNA level increased secondarily 5 weeks after the shunt closure.

possible therapeutic targets to treat this overflow-induced vasculopathy, we first investigated the mechanism of changes in pulmonary vascular wall thickness after shunt establishment and closure.

In patients with CTEPH, Moser and Braunwald ${ }^{6}$ had already reported marked structural changes of chronic $\mathrm{PH}$ in the open PAs. This high flow-induced vasculopathy is due to high pressure and high shear stress related to the redistribution of the cardiac outflow toward the unobstructed PA bed. ${ }^{7}$ This distal vasculopathy contributes as well as the proximal occlusion of PAs to the elevated pulmonary vascular resistance. Indeed, in our animal model, the overflow pulmonary vasculopathy, as induced by 5 -week aortopulmonary shunting, was associated with increased medial thickness of distal PAs related to SMC proliferation as showed by PCNA labeling. These findings are similar to the vascular remodeling reported in unobstructed territories of patients with CTEPH, ${ }^{6}$ suggesting thus that the overflow, with the increase of the shear stress that it induces, can be re- sponsible for the vascular remodeling. This remodeling is then associated with the progressive increase of PA resistances. We also found that 1 week after PA flow normalization, the SMC proliferation decreased whereas apoptosis, as assessed by TUNEL, increased markedly. This imbalance between SMC proliferation and apoptosis was not associated with alteration of vascular collagen accumulation.

High blood flow in the lungs was associated with an increase in ET-1 and ETA levels in lung tissue. ET-1 is a potent vasoconstrictor and mitotic peptide for vascular SMCs. ET-1 overexpression has been found in animal studies of flowinduced $\mathrm{PH}^{12-14}$ and in patients with primary $\mathrm{PH} .{ }^{17,18} \mathrm{ET}-1$ overproduction is probably a response to stimuli such as shear stress resulting from arterial pressure elevation. ${ }^{23}$ One week after shunt closure, lung ET-1 and ETA levels break, whereas PA remodeling still persists. However, 5 weeks after shunt closure, the normalization of PA wall thickness was associated with restoration of lung ET-1 synthesis. These data suggest that ET-1 may be involved in the

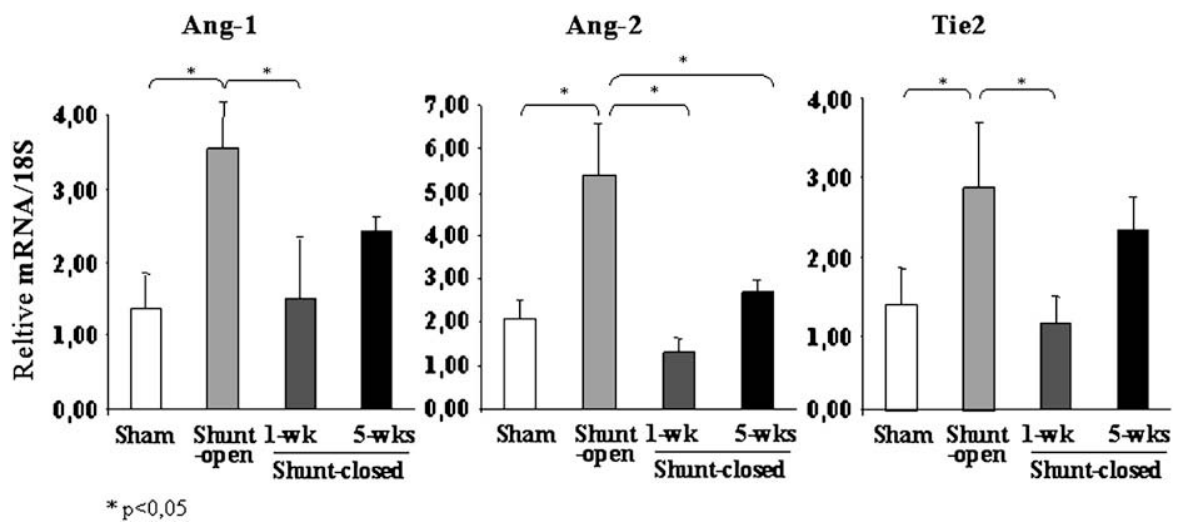

FIGURE 5. Quantification of angiopoietin-1, angiopoietin- 2 and Tie- 2 mRNA levels in the lung parenchyma from sham, shunt-open, and shunt-closed groups. High flow in the lungs induced an increase in angiopoietin-1, angiopoietin-2, and Tie-2 mRNA levels. Tie-2 mRNA level increased whereas the angiopoietin-1/angiopoietin-2 ratio remained the same. Shunt closure was followed by a decrease of expression of these genes. 
development and resolution of flow-induced lung vessel disease.

Rondelet and associates ${ }^{12}$ reported that ET- 1 inhibition by the ETA/ETB antagonist bosentan prevented pulmonary vascular remodeling in a piglet model of high flow-induced pulmonary vasculopathy. Interestingly, in our model, when flow was returned to normal by closing the shunt, ETB overexpression in lung tissue occurred, whereas ETA expression returned to sham values. ETB is selectively found in the distal PA bed ${ }^{24}$ both on SMCs and on endothelial cells. ${ }^{25} \mathrm{SMC}$ ETB mediates the same signals as ETA, inducing vasoconstriction and mitosis. In contrast, endothelial ETB serves as a clearance receptor for ET-1 and modulates ET-1 expression via a negative feedback loop and via the release of vessel relaxants such as nitric oxide. ${ }^{23}$ As the maximal contraction of PA ring induced by ET-1 was significantly lower in the 5-week shunt-closed group than in the sham group, ETB overexpression after shunt closure may reflect overexpression of the endothelial subtype rather than of the SMC subtype. ETB and ET-1 expressions follow the same evolution. This suggests that ETB may play a role in the control of ET-1 clearance and consequently may contribute to the regression of the distal arterial abnormalities induced by high flow.

In our study, eNOS RNA expression increased in the shunt-open group because of chronically increased shear stress, whereas endothelium-dependent relaxation of isolated pulmonary rings, which reflects nitric oxide production, was impaired. Of several studies investigating high-flow $\mathrm{PH}$ and eNOS expression, ${ }^{12,15,26-28}$ only one ${ }^{15}$ found increased eNOS protein levels; this increase was not found in lung homogenate but instead in fifth-generation isolated PA cells. In our model, the decrease in endothelium-dependent relaxation suggests that flow-induced PH may be related to alteration of the nitric oxide/cyclic guanosine monophosphate pathway. To investigate this possibility, we measured phosphodiesterase 5 activity in lung tissue from piglets with open aortopulmonary shunt. We found no change in phosphodiesterase 5 activity. Rondelet and associates ${ }^{29}$ reported that the phosphodiesterase 5 inhibitor seldinafil induced a partial improvement in flow-induced PH in piglets. These results suggest that alteration of the nitric oxide/cyclic guanosine monophosphate pathway may result from deterioration in eNOS activity and/or in nitric oxide stability. After shunt closure, endothelium-dependent relaxation of isolated PA rings returned gradually to normal, indicating improved matching of nitric oxide production to the number of SMCs.

Angiopoietin-1 is a peptide that contributes to lung vessel development by recruiting and promoting the division of vascular SMCs. ${ }^{19}$ Angiopoietin-1 and Tie-2 overexpressions were found in lung parenchyma from patients with PH. ${ }^{19}$ Thus, angiopoietin-1 may contribute to the excessive muscularization of distal PAs that characterizes PH. In our study, angiopoietin-1 and Tie-2 expressions increased after shunt opening and decreased after shunt closure. However, angiopoietin-2 expression followed angiopoietin-1 and Tie-2 expressions. Indeed, angiopoietin-2 blocks the angiopoietin-1/Tie-2 signal, resulting in a loosening of the tight vascular structure. ${ }^{30}$ Altough angiopoietin-2 expression increased in the shunt-open group and then decreased after the closure of the shunt, the rate angiopoietin-1/angiopoietin-2 remained the same in the four groups whereas Tie-2 was overexpressed in the shunt-open group and returned to normal in the shunt-closed groups. Thus, our results support that angiopoietin pathway may contribute to this vascular remodeling.

\section{CONCLUSION}

Flow-induced PA disease is associated with the increase of ET-1 and Tie-2 expressions in the lung parenchyma. Shunt closure to replicate the effects of PTE on nonobstructed lung territories was followed by normalization of ET-1 and Tie-2 expression and then gradually by resolution of the arterial abnormalities. These peptides hold promise as targets for treatments designed to improve PA disease in patients with CTEPH at risk of persistent PH after PTE.

\section{References}

1. Riedel M, Stanek V, Widimsky J, Prerovsky I. Long-term follow-up of patients with pulmonary thromboembolism. Late prognosis and evolution of hemodynamic and respiratory data. Chest. 1982;81:151-8.

2. Pengo V, Lensing AW, Prins MH, Marchiori A, Davidson BL, Tiozzo F, et al. Thromboembolic Pulmonary Hypertension Study Group. Incidence of chronic thromboembolic pulmonary hypertension after pulmonary embolism. $N$ Engl J Med. 2004;350:2257-64.

3. Jamieson SW, Kapelanski DP, Sakakibara N, Manecke GR, Thistlethwaite PA, Kerr KM, et al. Pulmonary endarterectomy: experience and lessons learned in 1,500 cases. Ann Thorac Surg. 2003;76:1457-62.

4. Dartevelle P, Fadel E, Chapelier A, Macchiarini P, Cerrina J, Parquin F, et al. Angioscopic video-assisted pulmonary endarterectomy for post-embolic pulmonary hypertension. Eur J Cardiothorac Surg. 1999;16:38-43.

5. Hartz RS, Byrne JG, Levitsky S, Park J, Rich S. Predictors of mortality in pulmonary thromboendarterectomy. Ann Thorac Surg. 1996;62:1255-9.

6. Moser KM, Braunwald NS. Successful surgical intervention in severe chronic thromboembolic pulmonary hypertension. Chest. 1973;64:29-35.

7. Hoeper MM, Mayer E, Simonneau G, Rubin LJ. Chronic thromboembolic pulmonary hypertension. Circulation. 2006;113:2011-20.

8. Peacock A, Simonneau G, Rubin L. Controversies, uncertainties and future research on the treatment of chronic thromboembolic pulmonary hypertension. Proc Am Thorac Soc. 2006;3:608-14.

9. Moser KM, Bloor CM. Pulmonary vascular lesions occurring in patients with chronic major vessel thromboembolic pulmonary hypertension. Chest. 1993;103:685-92.

10. Anderson EG, Simon G, Reid L. Primary and thromboembolic pulmonary hypertension: a quantitative pathological study. J Pathol. 1972;110:273-93.

11. Heath D, Edwards JE. The pathology of hypertensive pulmonary vascular disease: a description of six grades of structural changes in the pulmonary arteries with special reference to congenital cardiac septal defects. Circulation. 1958;18:533-47.

12. Rondelet B, Kerbaul F, Motte S, van Beneden R, Remmelink M, Brimioulle S et al. Bosentan for the prevention of overcirculation-induced experimental pulmonary arterial hypertension. Circulation. 2003;107:1329-35.

13. Reddy VM, Wong J, Liddicoat JR, Johengen M, Chang R, Fineman JR. Altered endothelium-dependent responses in lambs with pulmonary hypertension and increased pulmonary blood flow. Am J Physiol. 1996;271:H562-70.

14. Ovadia B, Reinhartz O, Fitzgerald R, Bekker JM, Johengen MJ, Azakie A, et al. Alterations in ET-1, not nitric oxide, in 1-week-old lambs with increased pulmonary blood flow. Am J Physiol Heart Circ Physiol. 2003;284:H480-90. 
15. Black SM, Fineman JR, Steinhorn RH, Bristow J, Soifer SJ. Increased endothelial NOS in lambs with increased pulmonary blood flow and pulmonary hypertension. Am J Physiol. 1998;275:H1643-51.

16. Nagaya N, Sasaki N, Ando M, Ogino H, Sakamaki F, Kyotani S, et al. Prostacyclin therapy before pulmonary thromboendarterectomy in patients with chronic thromboembolic pulmonary hypertension. Chest. 2003;123:338-43.

17. Giaid A, Yanagisawa M, Langleben D, Michel RP, Levy R, Shennib H, et al. Expression of endothelin-1 in the lungs of patients with pulmonary hypertension. N Engl J Med. 1993;328:1732-9.

18. Michel RP, Langleben D, Dupuis J. The endothelin system in pulmonary hypertension. Can J Physiol Pharmacol. 2003;81:542-54.

19. Thistlethwaite PA, Lee SH, Du LL, Wolf PL, Sullivan C, Pradhan S, et al. Human angiopoietin gene expression is a marker for severity of pulmonary hypertension in patients undergoing pulmonary thromboendarterectomy. J Thorac Cardiovasc Surg. 2001;122:65-73.

20. Rendas A, Lennox S, Reid L. Aorta-pulmonary shunts in growing pigs: functional and structural assessment of the changes in the pulmonary circulation. J Thorac Cardiovasc Surg. 1979;77:109-18.

21. Fadel E, Mazmanian GM, Baudet B, Detruit H, Verhoye JP, Cron J, et al. Endothelial nitric oxide synthase function in pig lung after chronic pulmonary artery obstruction. Am J Respir Crit Care Med. 2000;162:1429-34.

22. Appleman MM, Thompson WJ, Russell TR. Cyclic nucleotide phosphodiesterases. Adv Cyclic Nucleotide Res. 1973;3:65-98.
23. Galie N, Manes A, Branzi A. The endothelin system in pulmonary arterial hypertension. Cardiovasc Res. 2004;61:227-37.

24. Black SM, Mata-Greenwood E, Dettman RW, Ovadia B, Fitzgerald RK, Reinhartz O, et al. Emergence of smooth muscle cell endothelin B-mediated vasoconstriction in lambs with experimental congenital heart disease and increased pulmonary blood flow. Circulation. 2003;108:1646-54.

25. Bauer M, Wilkens H, Langer F, Schneider SO, Lausberg H, Schafers HJ. Selective upregulation of endothelin $\mathrm{B}$ receptor gene expression in severe pulmonary hypertension. Circulation. 2002;105:1034-6.

26. Fike CD, Kaplowitz MR, Bousamra M 2nd. eNOS and prostanoid enzymes in lungs of newborn piglets with chronic aortopulmonary shunts. Am J Physiol Lung Cell Mol Physiol. 2001;281:L475-82.

27. Parviz M, Bousamra M 2nd, Chammas JH, Birks EK, Presberg KW, Jacobs ER, et al. Effects of chronic pulmonary overcirculation on pulmonary vasomotor tone. Ann Thorac Surg. 1999;67:522-7.

28. Everett AD, Le Cras TD, Xue C, Johns RA. eNOS expression is not altered in pulmonary vascular remodeling due to increased pulmonary blood flow. Am J Physiol. 1998;274:L1058-65.

29. Rondelet B, Kerbaul F, Van Beneden R, Motte S, Fesler P, Hubloue I, et al. Signaling molecules in overcirculation-induced pulmonary hypertension in piglets: effects of sildenafil therapy. Circulation. 2004;110:2220-5.

30. Hanahan D. Signaling vascular morphogenesis and maintenance. Science. 1997; 277(5322):48-50 